\title{
Review
}

\section{Noninvasive Imaging of Peripheral Nerves}

\author{
Gautam Rangavajla Nassir Mokarram Nazanin Masoodzadehgan \\ S. Balakrishna Pai Ravi V. Bellamkonda \\ Wallace H. Coulter Department of Biomedical Engineering, Georgia Institute of Technology and \\ Emory University, Atlanta, Ga., USA
}

\section{Key Words}

Peripheral nervous system · Magnetic resonance imaging ·

Diffusion tensor imaging · Positron emission tomography .

Ultrasound also discussed, including innovative contrast agents, some of which enable multimodal imaging approaches and present opportunities for treatment application.

(c) 2015 S. Karger AG, Basel

\section{Introduction}

The integrity of the peripheral nervous system is crucial for a myriad of neuromuscular functions, including motor control, bladder control and sensory reception.

\section{Abbreviations used in this paper}

ADC apparent diffusion coefficient

CMAP compound muscle action potential

DTI diffusion tensor imaging

${ }^{18} \mathrm{~F} \quad$ fluorine-18

FA fractional anisotropy

FDG fluorodeoxyglucose

FMT fluorescence molecular tomography

GD-DTPA gadolinium diethylenetriaminepentacetate

Gf gadofluorine

MRI magnetic resonance imaging

PET positron emission tomography

PFC perfluorocarbon

ROC receiver operating characteristic

US ultrasound to enhance and extend the abilities of imaging methods are

\section{KARGER}

E-Mail karger@karger.com

www.karger.com/cto
(C) 2015 S. Karger AG, Basel

$1422-6405 / 15 / 2001-0069 \$ 39.50 / 0$ 
Table 1. Advantages and disadvantages of imaging methods

\begin{tabular}{|c|c|c|}
\hline MRI & $\begin{array}{l}\text { - recognizes nerves rapidly after injury } \\
\text { - correlates with electrophysiological analysis } \\
\text { - visualizes macrophage activity during Wallerian } \\
\text { degeneration } \\
\text { - has a higher resolution than US }\end{array}$ & $\begin{array}{l}\text { - cannot examine long nerves in a single scan } \\
\text { - cannot determine the degree of nerve injury }\end{array}$ \\
\hline US & $\begin{array}{l}\text { - improves the accuracy of nerve block injections } \\
\text { - visualizes long nerves in a single scan }\end{array}$ & $\begin{array}{l}\text { - has a relatively poor resolution } \\
\text { - is highly operator dependent } \\
\text { - cannot visualize nerves without anatomical landmarks } \\
\text { or nerves under bone }\end{array}$ \\
\hline${ }^{18} \mathrm{~F}-\mathrm{FDG}$ PET & $\begin{array}{l}\text { - visualizes metabolism } \\
\text { - has a high sensitivity } \\
\text { - can be used for early detection of disease }\end{array}$ & $\begin{array}{l}\text { - requires local production of rapidly decaying imaging } \\
\text { agents } \\
\text { - cannot directly image a nerve }\end{array}$ \\
\hline
\end{tabular}

However, peripheral nerve maladies can impact this functionality and severely decrease a patient's quality of life. Peripheral neuropathy affects over fifteen million people, and patient recovery depends on accurately assessing the nerve damage [Sessions and Nickerson, 2014]. Precise determination of the extent of neuronal damage can enable better diagnoses and care, thereby leading to appropriate intervention and accelerating the recovery process. To date, most evaluation approaches have focused on functional evaluation of the nerve state, providing little to no insight into the dynamics of damage or regenerative mechanisms. These functional electrophysiological tests may be complemented by invasive histological biopsies (such as sural nerve biopsies) [Malik et al., 2001]. However, nondestructive structural and functional assessment of peripheral nerves remains relatively unsophisticated and is therefore the focus of this review.

Noninvasive imaging techniques would ideally assess the neural structural and functional state with greater accuracy, rapid diagnoses, lower cost and minimal discomfort. Recent advances in the noninvasive imaging of peripheral nerves utilize both existing as well as novel techniques to not only visualize nerves, but also evaluate their structural integrity and metabolic activity. This review article addresses developments in magnetic resonance imaging (MRI), diffusion tensor imaging (DTI), ultrasound (US) and positron emission tomography (PET) in the con- text of peripheral nerve damage caused by lesions, neuropathies and ligation injuries through markers of nerve health and structure. The criteria used to compare the imaging methods include correlation with physiological and electrophysiological factors, ease of use, interoperator consistency and resolution. The unique capabilities of each modality are summarized in table 1 and explored in further detail in the sections below. The sections are subdivided by imaging method, and discuss current research in the field as well as priorities for future research with the aim of developing better clinical tools for peripheral nerve imaging.

\section{Magnetic Resonance Imaging}

MRI is a well-established imaging technique that can be used to differentiate injured peripheral nerves from healthy nerves. MRI utilizes a strong magnetic field to create a baseline net magnetization in the tissue followed by a disruption of the magnetization with a brief radio pulse, changing its direction and decreasing its longitudinal magnitude. The tissue's return to baseline magnetization can be described by two time constants, T1 and T2, with T1 describing the time for the longitudinal magnitude to return to the baseline level and T2 describing the time for the magnetization to realign to its original direction. Noncontrast MRI imaging of peripheral nerves typ- 
ically utilizes T2-weighted scans, which depicts injury sites as hyperintense compared to other tissue, likely as a result of edema at the injury site, which affects the T2 value. In most clinical applications, the MRI magnetic field strength is relatively low - typically below $3 \mathrm{~T}$; however, higher field strengths can better delineate peripheral nerves by increasing the resolution and signal-tonoise ratio [Zaidman et al., 2013].

\section{Application of MRI to Nerve Imaging: Magnetic \\ Resonance Neurography}

MRI scans can accurately determine the location of a lesion, provide a high level of anatomical detail, and correlate with electrophysiological evaluation - the current gold standard for nerve injury evaluation [Stanisz et al., 2001; Lacour-Petit et al., 2003]. Peripheral nerves imaged through injury and regeneration with noncontrast enhanced T2weighted scanning initially displayed isointensity with surrounding soft tissue prior to injury. Upon injury, the T2weighted intensity increased as a result of edema near the injury site, but over the course of the regenerative process the hyperintensity gradually decreased to baseline levels, corresponding with nerve regeneration [Lacour-Petit et al., 2003; Bendszus et al., 2004; Wessig et al., 2008].

Bendszus et al. [2004] examined the electrophysiology of injured rat sciatic nerves during controlled periods of degeneration and regeneration following ligation. Following injury, compound muscle action potential (CMAP) and electromyography measurements sharply decreased. They then gradually increased over 7 weeks postinjury, paralleling histological evidence of regeneration. Similarly, the T2-weighted nerve signal intensity was initially similar to surrounding tissue (isointense), and increased sharply postinjury before gradually decreasing over the regeneration period. This corresponds to the decrease in intensity corresponding to the first voluntary movements recorded by the electromyography. These results suggest that MRI can provide an accurate nerve injury assessment, allowing for an alternative, noninvasive measurement technique in cases where direct electrophysiological applications are not practical [Evans and Manji, 2013].

Although MRI represents a common clinical approach to nerve visualization, there is currently a trade-off between MRI resolution and the field of view, which inhibits accurate visualization of long nerves [Lacour-Petit et al., 2003]. Also, the technique cannot at present resolve the degree of injury, which is necessary for planning a course of surgery [Bendszus et al., 2004]. However, this can change as future research in MRI of peripheral nerves focuses on accurately determining the location and de- gree of nerve injury, thereby leading to more reliable diagnoses. New developments in contrast agents that suggest possible methods of locating the injury are promising areas of further investigation.

\section{Application of MRI to Nerve Imaging: DTI}

MRI can also be used to track the diffusion of water molecules in a technique known as DTI [Sheikh, 2010]. DTI imaging relies on analyzing metrics of nerve health, calculated from water diffusion, to create a numerical approach to understand nerve injury, since the diffusion patterns differ between healthy and injured nerves. As a numerical technique, DTI metrics can be compiled and analyzed, yielding statistical correlations with electrophysiology and histology, and improving diagnoses. These metrics include the tensor eigenvalues for parallel and perpendicular diffusion $\left(\lambda_{\|}\right.$and $\left.\lambda_{\perp}\right)$, as well as fractional anisotropy (FA) and apparent diffusion coefficient (ADC) [Lehmann et al., 2010; Morisaki et al., 2011; Guggenberger et al., 2012]. The eigenvalues are directly correlated with the amount of water diffusion either parallel or perpendicular to the nerve, and FA and ADC are composite values calculated from both $\lambda_{\|}$and $\lambda_{\perp}$. As relatively linear structures, healthy nerves maintain anisotropic diffusion as the water molecules are effectively restricted to one line of motion. After structural damage, however, the water molecules are more capable of diffusing orthogonal to the nerve, thereby decreasing the diffusion anisotropy.

Multiple studies demonstrate the validity of DTI diffusion metrics in characterizing nerve injury through correlation with established methods, such as electrophysiology and histology. Among these metrics, the FA values consistently respond to nerve injury and consistently correlate with histological markers of nerve health [Kakuda et al., 2011; Morisaki et al., 2011; Manganaro et al., 2014]. Studies in both human patients and animal models demonstrate that the FA values are significantly lower in injured nerves than in healthy nerves, due to the decrease in myelination and anisotropic diffusion [Kakuda et al., 2011; Morisaki et al., 2011; Chhabra et al., 2013; Mathys et al., 2013]. Similar to measurements of MRI hyperintensity, these values changed over the course of nerve structural regeneration, increasing as anisotropic water diffusion was restored.

FA values also correlate with the electrophysiological assessment of nerves, which is essential to demonstrate the clinical applicability of DTI. In clinical studies, patients maintain a significant positive correlation between the amplitude of the tibial nerve action potential and FA $(\mathrm{R}=0.86, \mathrm{p}=0.04)$ and between FA and CMAP measure- 
ments [Kakuda et al., 2011; Mathys et al., 2013]. The significant correlation between FA and CMAP assessments elevates DTI to the current gold standard in evaluating nerve health. This can serve to improve prediction of nerve regeneration in situations not conducive to direct electrophysiological measurement. Other DTI metrics also displayed correlations with aspects of nerve health [Lehmann et al., 2010; Morisaki et al., 2011; Guggenberger et al., 2012]. Of note, ADC demonstrates the ability to distinguish malignant nerve tumors and tumor-like lesions from benign lesions [Guggenberger et al., 2012; Chhabra et al., 2013]. However, results relating to these other DTI parameters contradict in their ability to characterize nerve structural integrity, possibly due to varying equipment, interoperator differences, patient variability or inherent properties of $\lambda_{\perp}, \lambda_{\|}$and ADC [Kakuda et al., 2011]. These factors, however, may have clinical relevance toward other aspects of nerve health, similar to ADC's ability to detect malignancy. Further investigation is necessary to understand this inconsistency as well as to develop technology or protocols to reduce variability, and perhaps discover other applications for these metrics.

DTI measurements can assess nerve health at various stages of recovery and to a high degree of accuracy. The Wallerian degeneration (fig. 1) following nerve injury was characterized through significant changes in measurement parameters, as was the resulting recovery process [Lehmann et al., 2010; Morisaki et al., 2011]. Of particular interest in clinical applications is to determine diagnostic thresholds for DTI parameters that correspond to the existence or lack of nerve damage for a particular neuropathy [Guggenberger et al., 2012; Mathys et al., 2013]. ROC (receiver operating characteristic) analysis and threshold calculation were used to evaluate the use of FA and ADC in studying carpal tunnel syndrome, and a threshold of FA in the median nerve lower than 0.47 or ADC above $1.054 \times 10^{-3}$ was considered an indicator of carpal tunnel syndrome [Guggenberger et al., 2012]. This method yielded high sensitivities ( 0.83 and 0.83 , respectively) and moderate specificities ( 0.67 and 0.54 , respectively) for FA and ADC. Similarly, the analysis of patients with polyneuropathy revealed an optimal FA diagnosing threshold of 0.514 , with ROC analysis stating a high sensitivity (0.73) and specificity (0.70) [Mathys et al., 2013]. Threshold analysis has a unique advantage over numerical-based imaging techniques, such as DTI and molecular imaging, and can improve diagnostic potential. Evidenced by the high sensitivity and specificity of the test after threshold analysis, this approach can provide common standards by which to gauge the extent of nerve injury.
As an MRI-based technique, DTI has a low spatial resolution (1.6-3 $\mathrm{mm}$ voxel dimensions) and, thus, attempts to acquire high-resolution scans may require an inconvenient amount of time [Jaermann et al., 2004; Lehmann et al., 2010; Kakuda et al., 2011; Manganaro et al., 2014]. Stronger magnets can help alleviate this issue, but further research should explore the development of a rapid imaging protocol for DTI scans [Kakuda et al., 2011].

\section{Ultrasonography}

US imaging relies on the echo pattern of high-frequency sound waves (5-15 MHz) generated by piezoelectric crystals to visualize peripheral nerves [Martinoli et al., 1996]. US imaging of nerves can be axial, with the ability to view fascicles, or along the long-axis, parallel to the nerve and visualizing the entire structure in one view. Nerves, which typically appear as hypoechoic areas, are typically distinguished from blood vessels by the presence of a fascicular pattern on axial scans [Martinoli et al., 1996; Kara et al., 2012].

As MRI in general provides a greater resolution and contrast scan of peripheral nerves, US imaging serves as a supporting tool for conducting other procedures [Martinoli et al., 1996; Walker et al., 2004; Marhofer and Chan, 2007; McCartney et al., 2007; Koscielniak-Nielsen et al., 2008]. US imaging currently supports nerve blocks in which a catheter is guided to the nerve. The catheter is used to inject anesthetics and provide pain relief. In these situations, US can provide physicians, who previously were limited to 'blind' injection or injection based solely on surface features, with a visualization of the target nerve within the tissue [McCartney et al., 2007; KoscielniakNielsen et al., 2008]. McCartney et al. [2007] evaluated the applicability of US in visualizing the median and ulnar nerves in the forearm. US in healthy volunteers suggested that these nerves can be visualized consistently from the elbow to the wrist, at a depth of up to $16.8 \mathrm{~mm}$ below the surface of the skin. In a clinical study of US-guided nerve blocks using a long-axis continuous US scan (5-13 MHz), the target nerve and catheter advance could be observed and resulted in successful nerve block administration [Koscielniak-Nielsen et al., 2008]. Further research is required to advance these techniques further and improve the delivery of nerve blocks. This allows for more reliable analgesia application for patients, especially as there are few, if any, complications from a US-guided nerve block [Marhofer and Chan, 2007].
Rangavajla/Mokarram/Masoodzadehgan/ Pai/Bellamkonda 
Fig. 1. Wallerian degeneration and axonal regeneration after injury. a Uninjured nerve is associated with Schwann cells and resident (inactivated) macrophages. b Immediately after injury, denervated myelinating Schwann cells release their myelin. These nonmyelinating Schwann cells then proliferate and produce cytokines/trophic factors which, along with other soluble factors produced from injured axons, activate resident macrophages and lead to the recruitment of hematogenous macrophages. The activated macrophages clear myelin and axon debris efficiently, change phenotype and produce factors that facilitate Schwann cell migration and alignment, and axon regeneration [Mokarram et al., 2012]. c At the later time points, injured axons form a growth cone and begin to regenerate along aligned Schwann cells. d Successful regeneration would occur by axonal reinnervation to the peripheral target. The destruction of the neuronal structure is the physiological alteration that effects image visualization of the injury site. The lack of constraint posed by the degenerating neuron on the direction of water flow within the cell enables detection of injury using DTI. Also, Wallerian degeneration of an entire nerve leads to edema near the injury site, enabling visualization in magnetic resonance neurography. Additionally, the macrophages arriving at the injury site can phagocytize and transport contrast agents to the injury site, enabling contrast imaging. Figure reproduced with permission from the publisher.

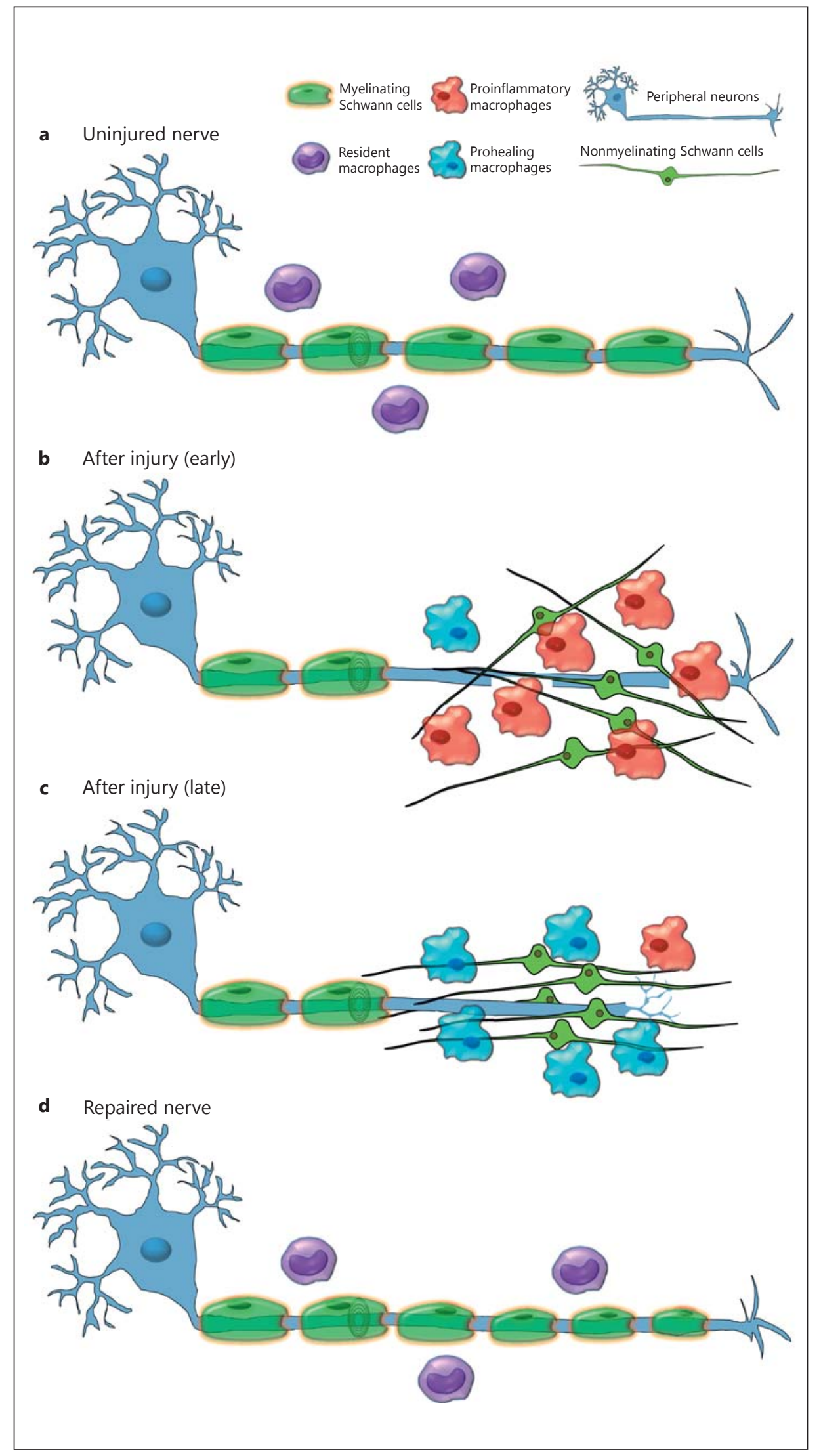


Certain US scans can resolve details as small as $400 \mu \mathrm{m}$ and can be combined with electrodiagnostic testing to yield improved diagnoses [Walker et al., 2004; Koenig et al., 2009; Kara et al., 2012]. US, unlike MRI, can visualize long nerves in a single study, but occasionally the nerve becomes difficult to follow due to either the nerve's anatomical location in terms of depth in the tissue or because of the lack of anatomical landmarks, at which point MRI may be preferable [Martinoli et al., 1996; McCartney et al., 2007; Kara et al., 2012]. In certain instances when visualizing and following nerves, US operators can have difficulty locating the nerve structure [McCartney et al., 2007]. Changes in local anatomy can alter the nerve layout and shape, complicating the scanning process and preventing the identification of vascular landmarks, thereby inhibiting the anatomical mapping.

Although most studies considered US to be inferior to MRI in resolution and contrast, Zaidman et al. [2013] noted greater sensitivity for US than MRI ( 0.93 and 0.67 , respectively) and equal specificities ( 0.86 for both), suggesting that US is preferable over MRI for visualizing peripheral nerve lesions. However, Padua and Hobson-Webb [2013] suggested in an editorial that these divergent results are primarily the product of operator bias, with the operator skill level playing a significant role in the outcome.

US imaging is highly dependent on the operator and is also affected by the age, gender, race and body position of the subject [Martinoli et al., 1996; Kara et al., 2012; Padua and Hobson-Webb, 2013]. Ongoing research into US imaging of peripheral nerves is validating its use and improving its ability to aid in performing nerve blocks or assisting in neurosurgery.

\section{Positron Emission Tomography}

Fluorine-18 $\left({ }^{18} \mathrm{~F}\right)$-fluorodeoxyglucose (FDG) PET imaging can determine regions of high metabolic activity through the use of a glucose molecule modified with an attached fluorine-18 ( $\left.{ }^{18} \mathrm{~F}-\mathrm{FDG}\right)$. Like glucose, FDG is localized and consumed in proportion to metabolic demand or activity. The ${ }^{18} \mathrm{~F}$ releases a positron that rapidly annihilates with an electron, producing $\gamma$ photons whose locations are determined by the PET imager and used to produce a map of the ${ }^{18} \mathrm{~F}-\mathrm{FDG}$. As a result of this mechanism, PET imaging has a very high sensitivity and is thus able to radiolabel the tissues with high glucose uptake.

The use of PET imaging in visualizing peripheral nerves is promising, yet it is in its infancy. Most of the current PET research in the peripheral nervous system involves evaluating peripheral nerve sheath tumors with very limited use in assessing injured peripheral nerves [Lucas et al., 1998; Ferner et al., 2000, 2008; Behera et al., 2011]. PET imaging has shown significant utility in detecting nerve sheath tumors as well as in other fields, and its value in evaluating peripheral nerve metabolism and nerve injury warrants further investigation [Osborn and Jaffer, 2009; Behera et al., 2011; Tatsumi et al., 2011]. The primary marker of nerve injury in ${ }^{18} \mathrm{~F}$-FDG PET is the increased uptake of ${ }^{18} \mathrm{~F}$-FDG by damaged nerves, which corresponds with higher levels of metabolic activity. As injured neurons maintain higher rates of neuron firing, the difference in the $\gamma$ photon emissions between injured and uninjured nerves can provide opportunities to locate and assess injured nerves [Behera et al., 2011]. Although current PET imaging of peripheral nerves uses metabolism to characterize injury, future research should seek to develop agents other than ${ }^{18} \mathrm{~F}-\mathrm{FDG}$ that specifically target injured nerves rather than only tissues with a high metabolism. As PET has a low spatial resolution, the coregistration of PET with a spatial imaging technique such as MRI or computed tomography allows for improved anatomic localization of the ${ }^{18} \mathrm{~F}-\mathrm{FDG}$ uptake [Behera et al., 2011; Tatsumi et al., 2011].

\section{Molecular Imaging}

PET and other molecular imaging techniques may be further from widespread clinical application than MRI, DTI and US, but they are likely to be valuable to the field of peripheral nerve imaging with further improvements in techniques. Similar to MRI analysis of immune activity and DTI analysis of microstructure, molecular imaging can provide detailed information on injury at a minute scale [Osborn and Jaffer, 2009; Behera et al., 2011]. Molecular imaging is inherently quantitative and shares with DTI the capability to develop diagnostic standards, statistical correlations and assess the degree of injury. Among molecular imaging techniques, fluorescencebased imaging has already shown results in the field of peripheral nerve imaging [Whitney et al., 2011]. Imaging methods such as fluorescence molecular tomography (FMT) may elaborate on this advancement to validate a molecular imaging technique to directly image peripheral nerves. FMT- or FMT-MRI-based imaging may view peripheral nerves independent of metabolic activity, and help create a foundation for new and improved diagnostic methods of peripheral nerve assessment.
Rangavajla/Mokarram/Masoodzadehgan/ Pai/Bellamkonda 


\section{Contrast Agents}

The addition of contrast agents before or during imaging can enhance or extend the functionalities of the nerve imaging methods detailed above [Ayyagari et al., 2006; Hahn et al., 2011]. In particular, MRI imaging can benefit from the inclusion of contrast agents.

Many MRI contrast agents enhance image contrast by altering either the $\mathrm{T} 1$ or $\mathrm{T} 2$ relaxation times, causing changes in signal intensity. Agents that target sites of nerve injury have been developed whose mechanisms of action typically include phagocytosis by macrophages that accumulate at the injury site [Bendszus and Stoll, 2003]. They can provide information on the macrophage response and insight into the general immune response during Wallerian degeneration and nerve regeneration. Following nerve injury, macrophages are recruited to the site to breakdown the distal end of the axon and the myelin sheaths (fig. 1). This destruction of the neuronal structure is the physiological alteration that affects image visualization of the injury site. The lack of constraint posed by the degenerating neuron on the direction of water flow enables detection of injury with DTI. Wallerian degeneration of an entire nerve will thus lead to edema near the injury site, enabling visualization in magnetic resonance neurography. Additionally, the macrophages arriving at the injury site during the degeneration phase of the process (fig. 1b) can phagocytize and transport contrast agents to the injury site, enabling contrast imaging, as discussed below.

Contrast-enhanced scans will improve upon noncontrast MRI scanning by highlighting immune activity in the postinjury axon [Bendszus and Stoll, 2003; Bendszus et al., 2005; Wessig et al., 2008; Stoll and Bendszus, 2009]. The development of perfluorocarbon (PFC) agents utilizing ${ }^{19} \mathrm{~F}$ presents an opportunity to pinpoint activity during Wallerian degeneration [Weise et al., 2011]. PFC is phagocytized by macrophages and transported to injury sites, where it accumulates and provides a means of visualizing immune activity. As ${ }^{19} \mathrm{~F}$ is not naturally present in the body, PFC allows for highly specific imaging of nerve injury sites.

Gadofluorine (Gf) particles also accumulate in sites undergoing Wallerian degeneration and cause increased contrast in T1-weighted scans [Bendszus et al., 2005]. The use of Gf-enhanced T1-weighted MRI was assessed in visualizing Wallerian degeneration in rat sciatic nerves after a crush injury [Bendszus et al., 2005]. In multiple studies, Gf injection soon after injury resulted in long-lasting T1-weighted contrast enhancement at the site of nerve

Noninvasive Imaging injury. Although visible in injured nerves, Gf-enhanced contrast is absent in healthy or regenerated nerves, and thus can assist in characterizing injured nerves relative to healthy nerves. Unlike gadolinium-DTPA, a conventional gadolinium-based contrast agent, Gf-enhanced T1weighted scanning has also been shown to visualize demyelination, an aspect that can aid in the early diagnoses of peripheral neuropathies, many of which initially manifest as nerve demyelination [Wessig et al., 2007]. A study in a rat experimental model of Guillain-Barré syndrome revealed a relationship between Gf-enhanced MRI scanning and CMAP measurements, corroborating the existing correlation between MRI and electrophysiological measurements, and confirming the validity of Gf-enhanced MRI in assessing nerve injury [Stoll et al., 2006].

A myriad of nanoparticle-based contrast agents in development can potentially add new multimodal capabilities to the field of peripheral nerve imaging. For example, Gd-DTPA was encased in stealth liposomes forming a nanoparticle that was able to provide long-lasting contrast in MRI in the bloodstream [Ayyagari et al., 2006]. Due to their encasement within the liposomes, the gadolinium particles were unable to rapidly diffuse out of the vasculature, and thereby provided a greater enduring contrast. Multivalent and multimodal particles, capable of T2weighted MRI contrast, binding of targeting ligands and absorption of near-infrared radiation permit diagnostic techniques combining MRI with other, more sensitive imaging modalities to acquire a complete picture of biological processes such as nerve injury [Jin et al., 2010]. Similar multimodal imaging approaches have also been developed between MRI and US, and between computed tomography, MRI and optical imaging [Liu et al., 2011; Xing et al., 2012]. In addition to expanding imaging capabilities, nanoparticle-based contrast agents can assist in treatment by visualizing targeted drug delivery or even serving as a treatment by themselves [Jin et al., 2010; Liu et al., 2011]. As many of these innovative contrast agents are still in the preclinical stage of development, information about their potential side effects is not known. By comparison, however, currently used radiological contrast agents are generally safe and only rarely cause side effects such as hypersensitivity or nephropathy [Andreucci et al., 2014].

\section{Conclusions}

Regarding the specific imaging techniques discussed in this article, each has a unique visualization characteristic (fig. 2) that results in some benefits and drawbacks 

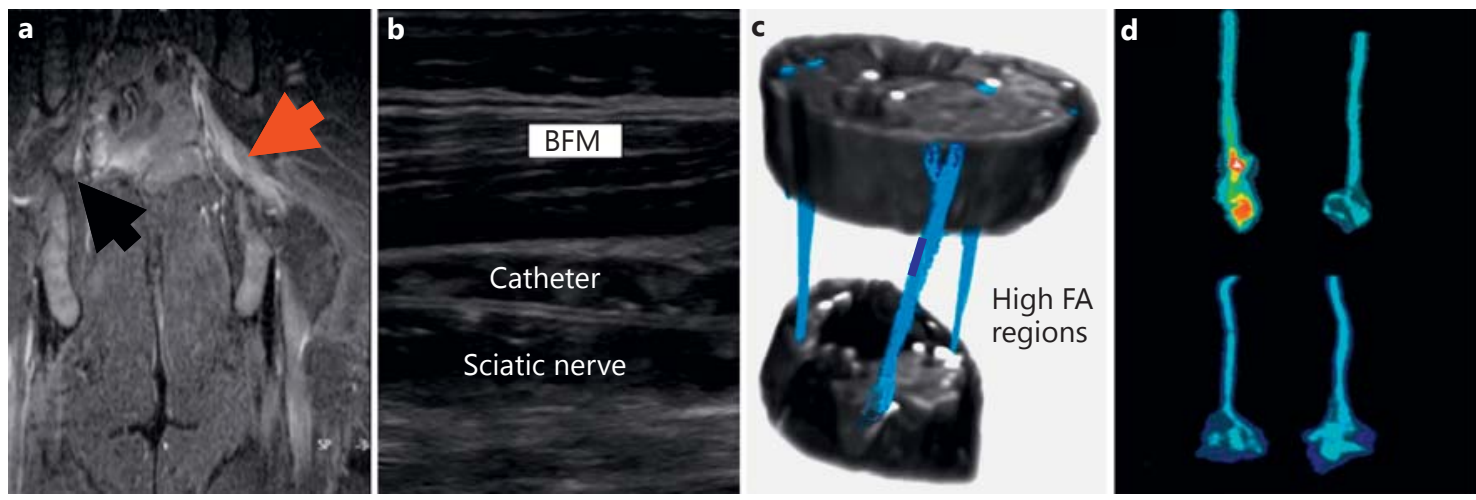

Fig. 2. Visualization of peripheral nerves. a MRI displays increased signaling from the injured nerve (red arrow) compared to the uninjured nerve (black arrow), which is not distinguishable from surrounding muscle [Lacour-Petit et al., 2003]. b US-guided nerve blocks allow visualization of the catheter, nerve and muscle (biceps femoris muscle, BFM) during procedures [Koscielniak-Nielsen et

(table 1). Thus, further research should be directed towards developing the comparative advantages of each method. Assessment by MRI achieves a high resolution in imaging both injured nerves with T2-weighted scans and Wallerian degeneration through contrast imaging. The ability to assess immune activity, however, is unique to MRI among the methods discussed, and thus must be included in further nerve imaging research. DTI, as a numerical technique, is best suited to general peripheral nerve analysis in clinical settings. The possibility of developing physiological standards on DTI measurements, the technique's strong correlation with electrophysiology across multiple nerve maladies and its ability to determine the degree of injury all add to its value as a tool for rapid and accurate diagnoses. US imaging in general is inferior to MRI in resolution and is highly situation dependent, precluding its current usage as a widespread primary assessment technique. However, the relative ease of use of US suggests a possible supporting role as a guiding tool for surgical or anesthetic procedures, though the reliability of the method needs improvement. Due to the limited information on PET imaging of peripheral nerves, it is difficult to draw comparisons with the other techniques, except for the obvious comparative advantage of visualizing metabolism in nerves. Further research is required on several aspects of PET application to determine its clinical relevance.

It is important, however, that the tools to noninvasively probe nerve structure and function be expanded. Functional imaging through MRI, US and PET provides the be- al., 2008]. c FA values determined by DTI can locate nerves and nerve tractography (blue) through tissue [Sheikh et al., 2010]. d PET of injured nerves suggests higher metabolism due to injury in the experimental injured nerve (top left) than in the uninjured right nerve (top right) or the control group (bottom) [Behera et al., 2011]. Figure reproduced with permission from the publisher.

ginning of an exploration to address this need. While the emphasis of this article is on imaging modalities, it is likely that advances in novel contrast agents will significantly enhance the power of imaging methodologies to go beyond structure and probe function, as well as increase structural/ spatial resolution. Therefore, development of unique contrast agents to extend the capability of MRI, US and PET imaging to yield structural and functional information with higher spatial acuity is promising and poised to significantly extend noninvasive evaluation of nerve structure and function.

\section{Acknowledgments}

This work was supported by grants from the National Institutes of Health (NS44409, NS65109, 1R41NS06777-1).

References

Andreucci, M., R. Solomon, A. Tasanarong (2014) Side effects of radiographic contrast media: pathogenesis, risk factors, and prevention. Biomed Res Int 2014: 741018.

Ayyagari, A.L., X. Zhang, K.B. Ghaghada, A. Annapragada, X. Hu, R.V. Bellamkonda (2006) Long-circulating liposomal contrast agents for magnetic resonance imaging. Magn Reson Med 55: 1023-1029.

Behera, D., K.E. Jacobs, S. Behera, J. Rosenberg, S. Biswal (2011) ${ }^{18} \mathrm{~F}$-FDG PET/MRI can be used to identify injured peripheral nerves in a model of neuropathic pain. J Nucl Med 52: 1308-1312.
Rangavajla/Mokarram/Masoodzadehgan/ Pai/Bellamkonda 
Bendszus, M., G. Stoll (2003) Caught in the act: in vivo mapping of macrophage infiltration in nerve injury by magnetic resonance imaging. J Neurosci 23: 10892-10896.

Bendszus, M., C. Wessig, A. Schutz, T. Horn, C. Kleinschnitz, C. Sommer, et al. (2005) Assessment of nerve degeneration by gadofluorine M-enhanced magnetic resonance imaging. Ann Neurol 57: 388-395.

-Bendszus, M., C. Wessig, L. Solymosi, K. Reiners, M. Koltzenburg (2004) MRI of peripheral nerve degeneration and regeneration: correlation with electrophysiology and histology. Exp Neurol 188: 171-177.

-Chhabra, A., R.S. Thakkar, G. Andreisek, M. Chalian, A.J. Belzberg, J. Blakeley, et al. (2013) Anatomic MR imaging and functional diffusion tensor imaging of peripheral nerve tumors and tumorlike conditions. AJNR Am J Neuroradiol 34: 802-807.

Evans, M., H. Manji (2013) Progress in peripheral nerve disease research in the last two years. J Neurol 260: 3188-3192.

-Ferner, R.E., J.F. Golding, M. Smith, E. Calonje, W. Jan, V. Sanjayanathan, et al. $(2008)\left[{ }^{18} \mathrm{~F}\right] 2-$ fluoro-2-deoxy-D-glucose positron emission tomography (FDG PET) as a diagnostic tool for neurofibromatosis 1 (NF1) associated malignant peripheral nerve sheath tumours (MPNSTs): a long-term clinical study. Ann Oncol 19: 390-394.

Ferner, R.E., J.D. Lucas, M. O’Doherty, R. Hughes, M. Smith, B. Cronin, et al. (2000) Evaluation of ${ }^{18}$ fluorodeoxyglucose positron emission tomography $\left({ }^{18} \mathrm{FDG}\right.$ PET $)$ in the detection of malignant peripheral nerve sheath tumors arising from within plexiform neurofibromas in neurofibromatosis 1. J Neurol Neurosurg Psychiatry 68: 353-357.

-Guggenberger, R., D. Markovic, P. Eppenberger, A. Chhabra, A. Schiller, D. Nanz, et al. (2012) Assessment of median nerve with MR neurography by using diffusion tensor imaging. Radiology 255: 194-203.

Hahn, M.A., A.K. Singh, P. Sharma, S.C. Brown, B.M. Moudgil (2011) Nanoparticles as contrast agents for in-vivo bioimaging: current status and future perspectives. Anal Bioanal Chem 399: 3-27.

-Jaermann, T., G. Crelier, K.P. Pruessmann, X. Golay, T. Netsch, A.M. van Muiswinkel, et al. (2004) SENSE-DTI at 3 T. Magn Reson Med 51: 230-236.

-Jin, Y., C. Jia, S.W. Huang, M. O’Donnell, X. Gao (2010) Multifunctional nanoparticles as coupled contrast agents. Nat Commun 1: 41.

Kakuda, T., H. Fukuda, K. Tanitame, M. Takasu, S. Date, K. Ochi, et al. (2011) Diffusion tensor imaging of peripheral nerve in patients with chronic inflammatory demyelinating polyradiculoneuropathy: a feasibility study. Neuroradiology 53: 955-960.

-Kara, M., L. Ozcakar, M. De Muynck, F. Tok, G. Vanderstraeten (2012) Musculoskeletal ultrasound for peripheral nerve lesions. Eur J Phys Rehabil Med 48: 665-674.
Koenig, R.W., M.T. Pedro, C.P. Heinen, T. Schmidt, H.P. Richter, G. Antoniadis, et al. (2009) High-resolution ultrasonography in evaluating peripheral nerve entrapment and trauma. Neurosurg Focus 26: E13.

Koscielniak-Nielsen, Z.J., H. Rasmussen, L. Hesselbjerg (2008) Long-axis ultrasound imaging of the nerves and advancement of perineural catheters under direct vision: a preliminary report of four cases. Reg Anesth Pain Med 33: 477-482.

Lacour-Petit, M.C., P. Lozeron, D. Ducreux (2003) MRI of peripheral nerve lesions of the lower limbs. Neuroradiology 45: 166-170.

Lehmann, H.C., J. Zhang, S. Mori, K.A. Sheikh (2010) Diffusion tensor imaging to assess axonal regeneration in peripheral nerves. Exp Neurol 223: 238-244.

Liu, Z., T. Lammers, J. Ehling, S. Fokong, J. Bornemann, F. Kiessling, et al. (2011) Iron oxide nanoparticle-containing microbubble composites as contrast agents for MR and ultrasound dual-modality imaging. Biomaterials 32: 6155-6163.

Lucas, J.D., M. O’Doherty, J. Wong, J. Bingham, P. McKee, J. Fletcher J, et al. (1998) Evaluation of fluorodeoxyglucose positron emission tomography in the management of soft-tissue sarcomas. J Bone Joint Surg Br 80: 441-447.

Malik R.A., A. Veves, D. Walker, I. Siddique, R.H. Lye, W. Schady, A.J. Boulton (2001) Sural nerve fibre pathology in diabetic patients with mild neuropathy: relationship to pain, quantitative sensory testing and peripheral nerve electrophysiology. Acta Neuropathol 101: 367-374.

Manganaro, L., M.G. Porpora, V. Vinci, S. Bernardo, P. Lodise, P. Sollazzo, et al. (2014) Diffusion tensor imaging and tractography to evaluate sacral nerve root abnormalities in endometriosis-related pain: a pilot study. Eur Radiol 24: 95-101.

Marhofer, P., V.W. Chan (2007) Ultrasoundguided regional anesthesia: current concepts and future trends. Anesth Analg 104: 12651269.

Martinoli, C., G. Serafini, S. Bianchi, M. Bertolotto, N, Gandolfo, L.E. Derchi (1996) Ultrasonography of Peripheral Nerves. J Peripher Nerv Syst 1: 169-178.

Mathys, C., J. Aissa, G.M. Zu Horste, D.C. Reichelt, G. Antoch, B. Turowski, et al. (2013) Peripheral neuropathy: assessment of proximal nerve integrity by diffusion tensor imaging (DTI). Muscle Nerve 48: 889-896.

McCartney, C.J., D. Xu, C. Constantinescu, S. Abbas, V.W. Chan (2007) Ultrasound examination of peripheral nerves in the forearm. Reg Anesth Pain Med 32: 434-439.

Mokarram, N., A. Merchant, V. Mukhatyar, G. Patel, R.V. Bellamkonda (2012) Effect of modulating macrophage phenotype on peripheral nerve repair. Biomaterials 33: 87938801.
Morisaki, S., Y. Kawai, M. Umeda, M. Nishi, R. Oda, H. Fujiwara, et al. (2011) In vivo assessment of peripheral nerve regeneration by diffusion tensor imaging. J Magn Reson Imaging 33: 535-542.

Osborn, E.A., F.A. Jaffer (2009) The year in molecular imaging. JACC Cardiovasc Imaging 2: 97-113.

Padua, L., L.D. Hobson-Webb (2013) Ultrasound as the first choice for peripheral nerve imaging? Neurology 80: 1626-1627.

Sessions, J., D.S. Nickerson (2014) Biologic basis of nerve decompression surgery for focal entrapments in diabetic peripheral neuropathy. J Diabetes Sci Technol 8: 412-418.

Sheikh, K.A. (2010) Non-invasive imaging of nerve regeneration. Exp Neurol 223: 72-76.

-Stanisz, G.J., R. Midha, C.A. Munro, R.M. Henkelman (2001) MR properties of rat sciatic nerve following trauma. Magnetic Reson Med 45: 415-420.

Stoll, G., M. Bendszus (2009) Imaging of inflammation in the peripheral and central nervous system by magnetic resonance imaging. Neuroscience 158: 1151-1160.

Stoll, G., C. Wessig, R. Gold, M. Bendszus (2006) Assessment of lesion evolution in experimental autoimmune neuritis by gadofluorine $\mathrm{M}$ enhanced MR neurography. Exp Neurol 197: 150-156.

Tatsumi, M., K. Isohashi, H. Onishi, M. Hori, T. Kim, I. Higuchi, et al. (2011) ${ }^{18}$ F-FDG PET/ MRI fusion in characterizing pancreatic tumors: comparison to PET/CT. Int J Clin Oncol 16: 408-415.

-Walker, F.O., M.S. Cartwright, E.R. Wiesler, J. Caress (2004) Ultrasound of nerve and muscle. Clin Neurophysiol 115: 495-507.

Weise, G., T.C. Basse-Luesebrink, C. Wessig, P.M. Jakob, G. Stoll (2011) In vivo imaging of inflammation in the peripheral nervous system by ${ }^{19}$ F MRI. Exp Neurol 229: 494-501.

Wessig, C., M. Bendszus, G. Stoll (2007) In vivo visualization of focal demyelination in peripheral nerves by gadofluorine $\mathrm{M}$-enhanced magnetic resonance imaging. Exp Neurol 204: 14-19.

Wessig, C., L. Jestaedt, M.W. Sereda, M. Bendszus, G. Stoll (2008) Gadofluorine M-enhanced magnetic resonance nerve imaging: comparison between acute inflammatory and chronic degenerative demyelination in rats. Exp Neurol 210: 137-143.

Whitney, M.A., J.L. Crisp, L.T. Nguyen, B. Friedman, L.A. Gross, P. Steinbach P, et al. (2011) Fluorescent peptides highlight peripheral nerves during surgery in mice. Nat Biotechnol 29: 352-356.

Xing, H., W. Bu, S. Zhang, X. Zheng, M. Li, F. Chen, et al. (2012) Multifunctional nanoprobes for upconversion fluorescence, MR and CT trimodal imaging. Biomaterials 33: 1079-1089.

Zaidman, C.M., M.J. Seelig, J.M. Baker, S.E. Mackinnon, A. Pestronk (2013) Detection of peripheral nerve pathology. Neurology 80: 1634-1640. 